\title{
Investigating the performance of an amplitude-independent algorithm for detecting the hand muscle activity of stroke survivors
}

\begin{abstract}
To make robotic hand devices controlled by surface electromyography (sEMG) signals feasible and practical tools for assisting patients with hand impairments, the problems that prevent these devices from being widely used have to be overcome. The most significant problem is the involuntary amplitude variation of the sEMG signals due to the movement of electrodes during forearm motion. Moreover, for patients who have had a stroke or another neurological disease, the muscle activity of the impaired hand is weak and has a low signal-to-noise ratio (SNR). Thus, muscle activity detection methods intended for controlling robotic hand devices should not depend mainly on the amplitude characteristics of the sEMG signal in the detection process, and they need to be more reliable for sEMG signals that have a low SNR. Since amplitudeindependent muscle activity detection methods meet these requirements, this paper investigates the performance of such a method on people who have had a stroke in terms of the detection of weak muscle activity and resistance to false alarms caused by the involuntary amplitude variation of sEMG signals; these two parameters are very important for achieving the reliable control of robotic hand devices intended for people with disabilities. A comparison between the performance of an amplitude-independent muscle activity detection algorithm and three amplitude-dependent algorithms was conducted by using sEMG signals recorded from six hemiparesis stroke survivors and from six healthy subjects. The results showed that the amplitude-independent algorithm performed better in terms of detecting weak muscle activity and resisting false alarms.
\end{abstract}

Keyword: Surface electromyography; Amplitude independent; Weak muscle activity detection; False alarms; Hemiparesis stroke survivors 